\title{
Effects of Field of View on Human Locomotion
}

\author{
Alexander Toet*, Marieke van der Hoeven, Mirela Kahrimanović, Nico J. Delleman \\ TNO Human Factors, P.O. Box 23, 3769 ZG Soesterberg, the Netherlands
}

\begin{abstract}
Field of view (FOV) restrictions are known to impair human performance for a range of different tasks. However, the effects of FOV restrictions on human locomotion through a complex environment are still not clear. This is particularly important for the development and deployment of FOV restricting devices like Head Mounted Displays (HMD's), which generally have FOV's that are much smaller than the unrestricted FOV. We investigated the effects of both horizontal and vertical FOV restrictions on the walking speed and head movements of participants manoeuvring through complex 3D obstacle courses. All FOV restrictions tested significantly increased the time needed to complete the courses, compared to the unrestricted condition. The time needed to traverse a course was significantly longer for a vertical FOV of $18^{\circ}$ than for a vertical FOV of $48^{\circ}$. For a fixed vertical FOV size, the traversal time was constant for horizontal FOV sizes ranging between $75^{\circ}$ and $180^{\circ}$, and increased significantly for the $30^{\circ}$ horizontal FOV condition. The implications of the current findings for the development of devices with FOV restrictions (like HMD's) are discussed.
\end{abstract}

Keywords: field-of-view, locomotion, maneuvering

\section{INTRODUCTION}

In this study we investigated the impact of instantaneous field-of-view restrictions on locomotion performance of participants manoeuvring through two different courses with horizontal and vertical obstacles. Several studies have shown that field-of-view restrictions degrade human observer performance for a range of different tasks ${ }^{1,3-6,8,12,13,20,21,23-25}$. However, the relationship between manoeuvring performance and field-of-view size is currently not fully known.

Head-mounted displays with field-of-views limited to $40^{\circ}-70^{\circ}$ are frequently deployed in virtual environments for training and rehearsing tasks involving human locomotion through complex environments (e.g. first responder actions, military operations in urban terrain), for the evaluation of designs (e.g. buildings, ships, factories), and for entertainment purposes (gaming). Dismounted soldiers performing night time operations in urban terrain frequently deploy nightvision goggles, with field-of-views that are typically limited to $30^{\circ}-40^{\circ}{ }^{17}$. In all these applications the field-of-view is considerably smaller than the unrestricted field-of-view, which has an average horizontal angle of approximately $200^{\circ}$, and an average vertical angle of about $135^{\circ}{ }^{26}$. Restricting the human visual field may compromise an observer's ability to control heading or process spatial information ${ }^{14}$, can influence distance estimates ${ }^{23}$, and compromises postural stability ${ }^{1,2,6,19}$. Most of the abovementioned tasks require the analysis of spatial relations between objects in the environment, the control of heading during locomotion through the environment, and the continuous maintenance of postural equilibrium. Any restriction of the peripheral visual field may therefore be detrimental for locomotion task performance. Increasing the amount of peripheral information by extending the field-of-view of head mounted displays and night vision goggles is costly, reduces their resolution or makes them heavier and therefore less comfortable to wear ${ }^{11}$. Moreover, in virtual environment applications, wider field-of-views yield greater sensations of motion sickness ${ }^{15,16}$. To determine a trade-off between human performance, cost, and ergonomic aspects we therefore need to know how field-of-view restrictions affect human locomotion through complex structured environments. In this study we characterised locomotion performance by the time participants needed to walk as fast as possible through two different obstacle courses while making as few errors as possible. Our hypothesis was that participants would need more time to complete the courses when their field-of-view was restricted.

* lex.toet@tno.nl; phone +31-346-356237; fax +31-346-353977; http://lextoet.googlepages.com 


\section{EXPERIMENT I: NAVIGATION THROUGH AN ARTIFICIAL FOREST}

This experiment was performed to investigate the effects of FOV restrictions on the speed and accuracy of participants performing a maneuvering task through a complex environment resembling an artificial forest.

\subsection{Method}

Tubular isolation material was used to create an indoor obstacle course resembling an artificial forest (Fig. 1). Approximately 90 'tree trunks' were evenly distributed over a large room. 'Branches' were horizontally attached to the 'trunks' at two different height intervals: (1) between ankle level and knee level, and (2) between waist and neck level. Traversing this obstacle course required participants to look both left-right (to avoid tree trunks) and up-down (to avoid tree branches). Broken black lines on the floor, marked with the letters $\mathrm{A}, \mathrm{B}, \mathrm{C}, \mathrm{D}, \mathrm{E}$, and $\mathrm{H}$, indicated six different routes of approximately equal length through the artificial forest.

A pilot goggle was used to restrict the field-of-view and simulate a night vision device. By fitting circular tubes with lengths of respectively 100,23 , and $0 \mathrm{~mm}$ (no tube(s)) to the openings of the goggle the field-ofview was restricted to respectively $20^{\circ}, 40^{\circ}$ and $60^{\circ}$ (Fig. 2). Night vision was simulated through a combination of two optical filters: a 139 Green filter and a 452 Sixteenth White Diffusion filter (www.leefilters.com). Monocular field-of-view conditions were obtained by fitting an opaque occluder to the goggle opening in front of the dominant eye.

The experiment consisted of 6 different viewing conditions: three FOV sizes $\left(20^{\circ}, 40^{\circ}\right.$, and $\left.60^{\circ}\right)$ and monocular as well as binocular view. The viewing conditions were counterbalanced across order, course and the combination course-goggles, using two different Greek-Latin squares ${ }^{22}$. Each participant performed the experiment for all 6 conditions. At the start of an experiment a participant first read the instructions. At the beginning of each trial the experimenter guided the participant to the start of one of the 6 possible routes. The experimenter then asked the participant to traverse the artificial forest as fast as possible along the assigned route, and to avoid touching any objects on their way. The participant then put on the goggles, and started walking after a verbal signal from the experimenter. The experimenter measured the time the participant needed to complete a route by means of a stopwatch. The visual acuity of the participants, with and without the different goggles, was measured between trials.
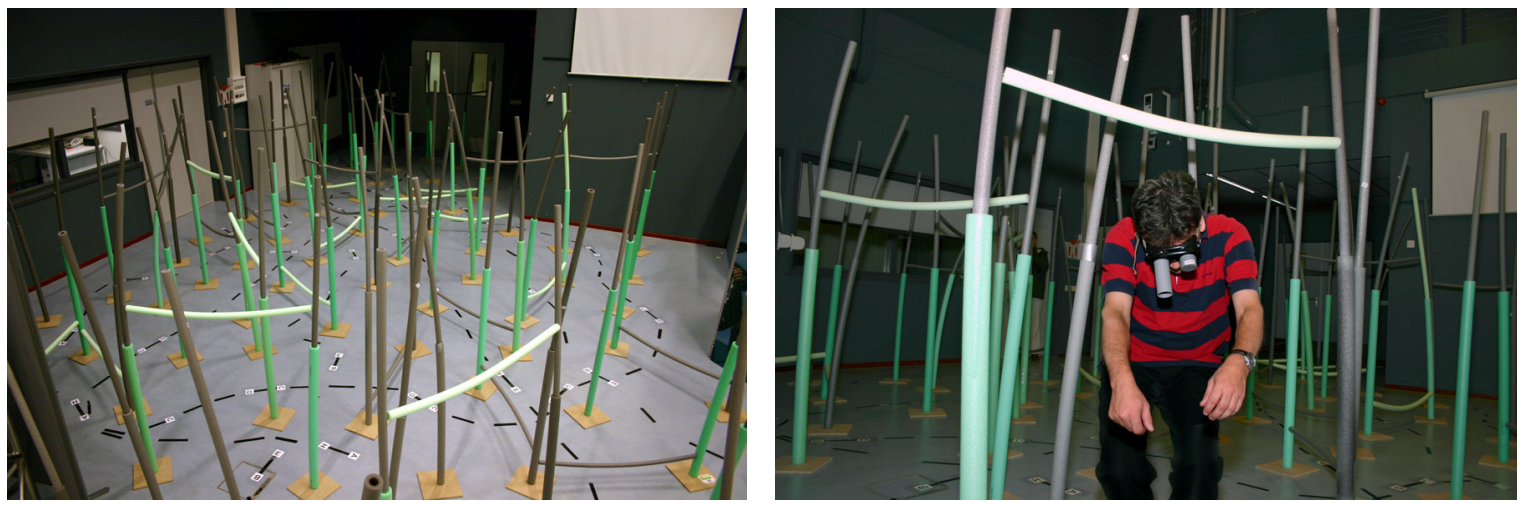

Fig. 1. Left: the artificial forest with horizontal and vertical obstructions. Right: participant traversing the forest and ducking to avoid a horizontal 'branch'. 
Proc. SPIE Vol. 6955-16, Head- and Helmet-Mounted Displays XIII: Design and Applications, Orlando FL, USA, March 2008
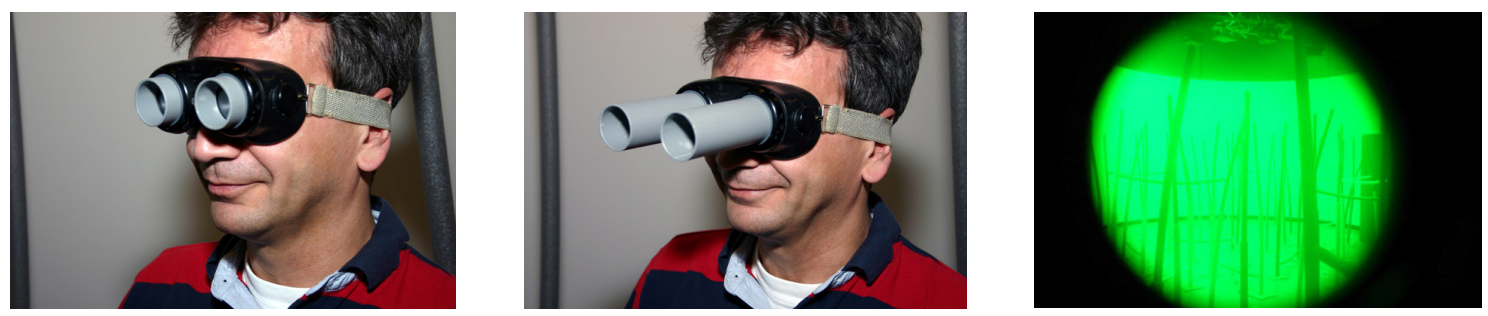

Fig. 2. Left, middle: Goggles equipped with tubes of different lengths to create different FOV sizes. Right: A green filter was used to simulate the view through a night vision goggle.

A total of 12 people (6 men and 6 women) ranging in age from 19 to 28 years participated in the experiment in return for monetary compensation. All participants had normal to corrected-to-normal (contact lenses) visual acuity, ranging from 0.5 to 2 , as established with the TNO visual acuity test, and were free from any impediments to normal locomotion as determined by self-report. Visual acuity with the restricting field-of-view spectacles ranged from 0.25 to 1 . The experimental protocol was approved by TNO Human Factors internal review board on experiments with human participants. The participants gave their informed consent prior to testing.

\subsection{Results}

Fig. 3 shows that, for each FOV size, the average time needed to traverse the artificial forest is consistently larger for the monocular viewing condition compared to the corresponding binocular viewing condition. For both viewing conditions, the time needed to traverse the obstacle course decreases with increasing field-of-view size. A repeated measure analysis of variance using Eyes (monocular and binocular), and FOV $\left(20^{\circ}, 40^{\circ}\right.$, and $\left.60^{\circ}\right)$ as factors indeed shows significant main effects of Eyes and FOV: $F(1,11)=91,326$ , $\mathrm{p}=.000$, and $\mathrm{F}(2,22)=111.813, \mathrm{p}=.000$, respectively. There is also an significant interaction between Eyes and FOV: $F(2,22)=4.909, p=.017$ (Fig. 3). This means that the absolute improvement of performance by using binocular vision increases for decreasing FOV sizes. When a similar analysis is performed on a logarithmic representation of the measured time data, the main effects of Eyes and FOV remain significant, but the interaction between Eyes and FOV is no longer significant. This implies that performance improves with a constant factor (1.26) at all FOV sizes. 
Proc. SPIE Vol. 6955-16, Head- and Helmet-Mounted Displays XIII: Design and Applications, Orlando FL, USA, March 2008

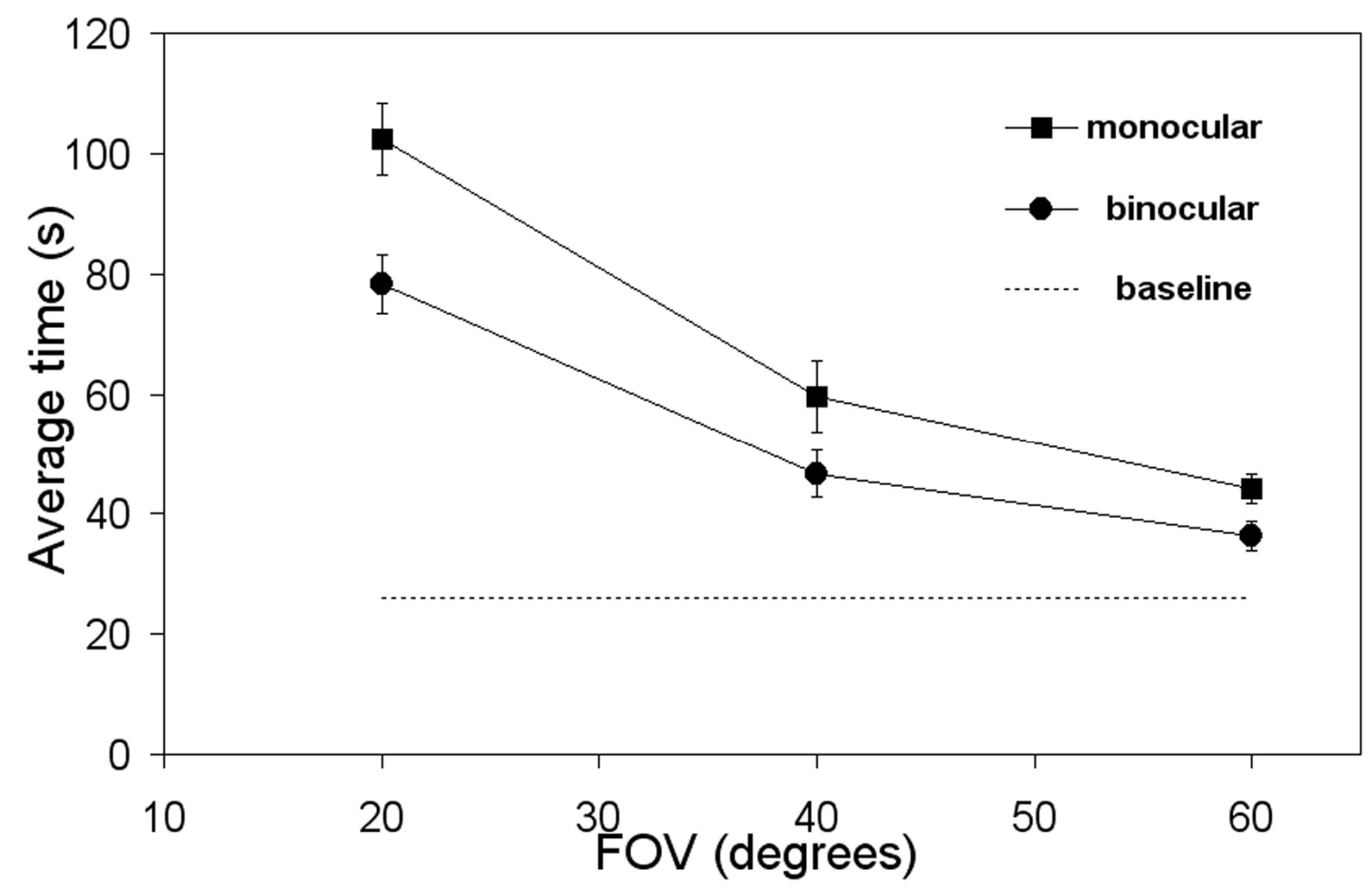

Fig. 3. The average time needed to traverse the artificial forest as a function of the field-of-view.

\section{EXPERIMENT II: TRAVERSAL OF AN OBSTACLE COURSE}

This experiment was performed to investigate the effects of FOV restrictions on the speed and accuracy of participants performing a maneuvering task through an obstacle course consisting of a straight corridor containing horizontal and vertical obstacles.

\subsection{Method}

The obstacle course was an 8 meter long and $135 \mathrm{~cm}$ wide straight walled course. The walls were made from wooden frames covered with white linen sheets. These walls simplified the visual environment, and eliminated the occurrence of visual distractions during the experiment.

Obstacles were placed at three different locations along the course, with a distance of $115 \mathrm{~cm}$ between the starting point and the first obstacle, $160 \mathrm{~cm}$ between two consecutive obstacles, and $115 \mathrm{~cm}$ between the last obstacle and the end of the course (Fig. 4). Each of these obstacles required the performance of different bodily movements in order to cross them. 
Proc. SPIE Vol. 6955-16, Head- and Helmet-Mounted Displays XIII: Design and Applications, Orlando

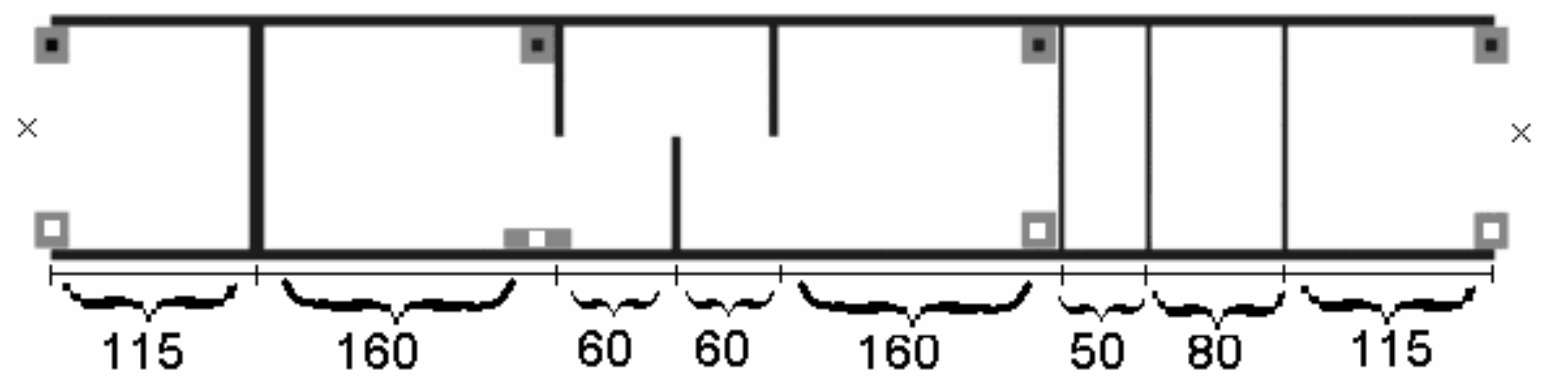

\section{- light sensor $\square$ light reflector}

\section{$\times$ starting/end point}

Fig. 4 Top view of the course with the three different obstacles (all dimensuions are given in $\mathrm{cm}$ ). From left to right: the bar, the three dividers and the three shelves. Light sensors and reflector were placed at 4 locations along the course, dividing it into 3 segments, with a length of respectively (left to right) 275 $\mathrm{cm}, 280 \mathrm{~cm}$, and $245 \mathrm{~cm}$.

One of the obstacles was a cardboard bar (Fig. 5), mounted such that its midpoint was always $110 \mathrm{~cm}$ above the ground, while the bar itself could either be placed in a horizontal or diagonal orientation. The bar was made from soft material (cardboard) to prevent participants from hurting themselves. This obstacle was located at a distance of $115 \mathrm{~cm}$ from the nearest entrance of the course. Participants had to duck underneath the bar to cross this segment of the course.

The second obstacle was placed in the middle of the course and consisted of three dividers made from the same material that was used to construct the walls of the course. Two dividers were attached perpendicular to one wall of the course, one behind the other with a distance of $120 \mathrm{~cm}$ between them. The third divider was attached perpendicular to the opposite course wall, such that it was located at the midpoint of the 120 $\mathrm{cm}$ interval defined by the first and last divider (Fig. 4). Thus, the separation between two consecutive dividers was $60 \mathrm{~cm}$, and the overall length of this obstacle was $180 \mathrm{~cm}$. To traverse this segment of the course participants had to follow an S-curved trajectory along the three dividers.

The third obstacle consisted of three thin wooden shelves, with heights of 20,30 and $40 \mathrm{~cm}$ respectively. The shelves were placed in an upright position on the ground, perpendicular to the walls. The distance between the first and the second shelf was $50 \mathrm{~cm}$, and the distance between the second and third shelf was $80 \mathrm{~cm}$, resulting in an overall obstacle length of $130 \mathrm{~cm}$. This obstacle was located at a distance of $115 \mathrm{~cm}$ from the nearest entrance of the course. The shelves were not rigidly fixed to the ground, to assure that any physical contact between a participant and the shelves would cause the shelves to displace or fall over. This was done to prevent participants from hurting themselves. To cross this obstacle participants had to step over each shelf (Fig. 5). 

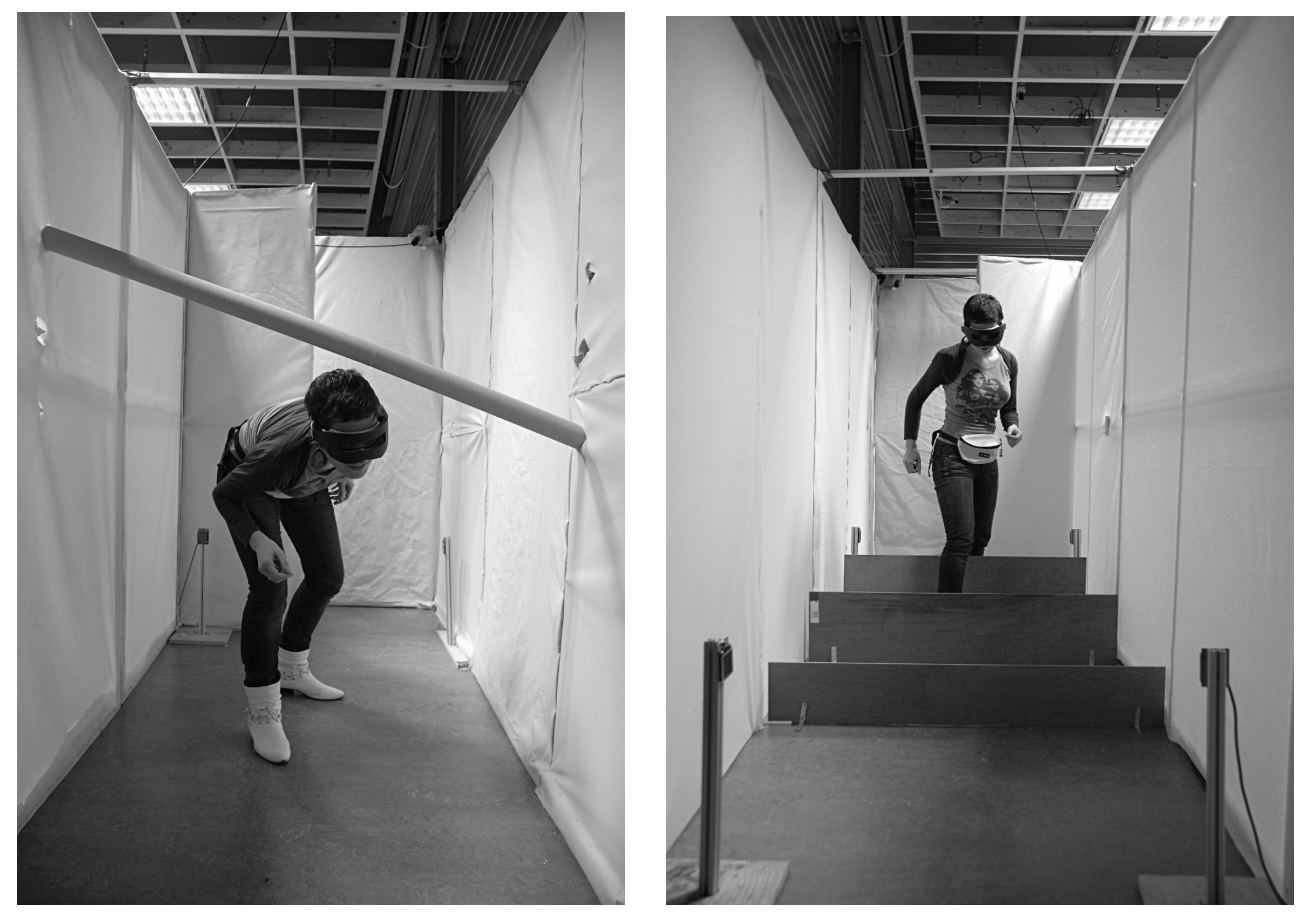

Fig. 5. The obstacle course. Left: participant ducking to avoid hitting the bar. Right: participant stepping over the shelves.

To register the time participants needed to traverse the entire course, and the time needed to cross each obstacle, we used 4 pairs of poles equipped with infra-red light-emitting-diodes, photoelectric beam sensors and retro reflectors (type Velleman PEM5D; www.velleman.be). The devices functioned by emitting and registering the return of an infrared light beam, which was reflected by a little mirror on its opposite pole. Whenever a participant interrupted a beam the moment of interruption was registered by the time acquisition system. One pair of poles was placed at the beginning and one at the end of the course. The third pair was placed just before the first divider of the second obstacle, when viewed from the side of the bar. Finally, the last pair of poles was placed just before the first shelf of the last obstacle, when viewed from the side of the three dividers (Fig. 4). Thus the course was divided into 3 segments: the bar-segment of length $275 \mathrm{~cm}$, the dividers-segment of length $280 \mathrm{~cm}$, and the shelves-segment of length $245 \mathrm{~cm}$.

A ski goggle from which the lenses had been removed was used to restrict the field-of-view. The different conditions of field-of-view restriction were achieved by attaching black cardboard masks with rectangular openings of different sizes to the goggle by means of Velcro tape (Fig. 6). The set of masks provided two vertical and seven horizontal field-of-view restrictions. The vertical field-of-view sizes were respectively $18^{\circ}$ and $48^{\circ}$, and the horizontal field-of-view sizes were respectively $30^{\circ}, 75^{\circ}, 112^{\circ}, 120^{\circ}, 140^{\circ}, 160^{\circ}$ and $180^{\circ}$.
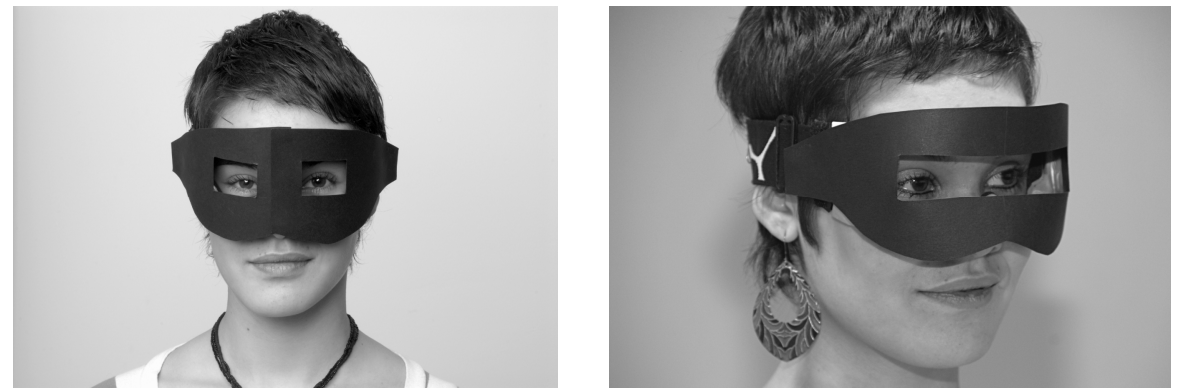

Fig. 6. Participant wearing the goggle with different field-of-view restricting masks. Left: $48^{\circ}$ vertical $/ 75^{\circ}$ horizontal. Right: $48^{\circ}$ vertical $/ 120^{\circ}$ horizontal. 
Proc. SPIE Vol. 6955-16, Head- and Helmet-Mounted Displays XIII: Design and Applications, Orlando FL, USA, March 2008

A 7 (horizontal field-of-view) x 2 (vertical field-of-view) x 3 (repeated measures) within participants design was used. To cancel possible learning effects, the different field-of-view conditions and the two different starting/end points were balanced over trials using a Latin square design ${ }^{22}$. In addition to the restricted field-of-view conditions, the full field-of-view condition was tested at the beginning and at the end of the experiment. This was done to check for a possible overall learning effect that may be caused by the fact that the participants get familiar with the course during the experiment. The dependent variable was the time needed to traverse the course.

A total of twelve paid participants, six males (mean age 21.8 years, standard deviation 2.2 years) and six females (mean age 21.2 years, standard deviation 2.1 years) participated in this study. The participants were all students, with normal (20/20) or corrected-to-normal (contact lenses) vision, and were free from any impediments to normal locomotion as determined by self-report. The experimental protocol was approved by TNO Human Factors internal review board on experiments with human participants. The participants gave their informed consent prior to testing.

First the participants were asked to fill out an informed consent form and a questionnaire. Then, they were given written and verbal instructions about the course, the three different obstacles, the goggle and the head movement detector. The participants were then asked to take place at a cross marked on the ground at one entrance of the course, and to stand still for 5 seconds with their head in an upright position, facing the entrance and looking straight ahead. After 5 seconds a start sign was given by the experimenter, and the participants had to traverse the course as fast as possible, but also as safely as possible (i.e. without hitting any part of the course). A cross on the opposite side of the course indicated the end of the trial, where the participants had to stop and again stand still in an upright position for 5 seconds.

At this stage there were two alternative return routes. The first option was to turn 180 degrees around and re-traverse the course from that point. The second option was to walk along a route on the outside of the obstacle course, back to the initial starting position, and to start re-traversing the course from that point. These two different walking routes were balanced over trials, to reduce possible learning effects. The experimenter indicated the new starting position after finishing each trial. In this way all the different fieldof-view conditions were tested, and each specific condition was repeated three times. Only the last two measurements were used for the data analysis, and the first one served as a practicing trial. Between the different field-of-view conditions there was a short period of rest during which the field-of-view restrictions were removed, to reduce possible transfer effects of the last condition on the following trial. During these breaks the participants had the opportunity to give their subjective impression about the effects of the restriction on their performance, and to express other feelings that might be relevant for the experiment.

To reduce possible learning effects (adaptation to the course), the three wooden shelves were randomly reordered every time the participant had completed seven trials, and the orientation of the bar was altered in a random order.

To compare the effects of the different field-of-view restrictions on the time variable a Repeated Measures ANOVA was performed, with horizontal restrictions (9) and vertical restrictions (2) as the within-subject factors. This was done for the entire course and also for every obstacle segment individually. If significant overall effects were found, a Paired Samples T-test was used to reveal pairwise differences ( $\alpha$ set to 5\%).

\subsection{Results}

The horizontal field of view had a significant main effect on the average time needed to traverse the entire course $(\mathrm{F}(8,88)=102.2, \mathrm{p}<0.01$; Fig.7). Also, a significant difference was found between the two vertical field-of-view restrictions $(\mathrm{F}(1,11)=21.0, \mathrm{p}<0.01)$.

A Paired Samples T-test revealed that, for most horizontal field-of-view restrictions, the two vertical fieldof-views had a significantly different effect on the average time needed to traverse the course, except for $75^{\circ}$ and $120^{\circ}$ horizontal field-of-view. Figure 7 shows that the average time needed to traverse the entire course was shorter with $48^{\circ}$ vertical field-of-view than with $18^{\circ}$. The post hoc test for the horizontal fieldof-view variable showed significant differences between the two full field-of-view conditions and all restricted field-of-view conditions $(\mathrm{p} \leq 0.01)$. The results were similar for both vertical field-of-views. Figure 7 illustrates that a restriction of the horizontal field-of-view yielded an increase in the average time needed to traverse the entire course. The same test also revealed a significant difference between the most 
Proc. SPIE Vol. 6955-16, Head- and Helmet-Mounted Displays XIII: Design and Applications, Orlando FL, USA, March 2008

restricted horizontal field-of-view condition $\left(30^{\circ}\right)$ and all other restricted horizontal field-of-view conditions: the most restricted horizontal condition yielded the largest average time $(\mathrm{p} \leq 0.01)$. No significant differences were found between the other restricted field-of-view conditions. Finally, there was no significant difference between the full field-of-view condition tested at the start of the experiment (datapoint U1 in Fig. 7) and the one tested at the end of the experiment (datapoint U2 in Fig. 7) meaning that there were no overall learning effects.

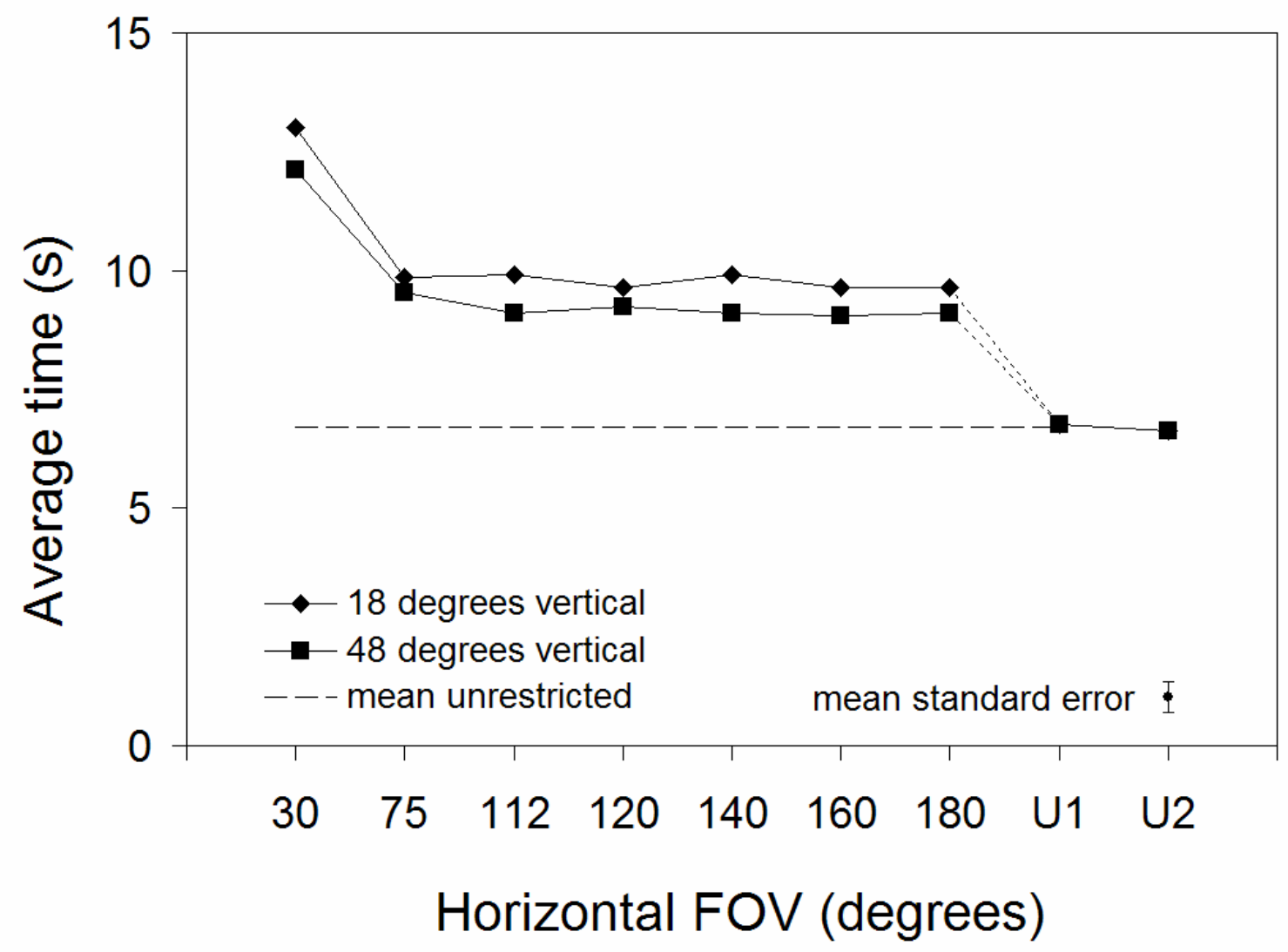

Fig. 7. The influence of field-of-view on the average time needed to traverse the entire course. U1 refers to the unrestricted field-of-view condition tested at the start of the experiment, and U2 indicates the same condition tested at the end of the experiment. The dashed line indicates the mean value of U1 and U2, and represents a performance baseline.

\section{DISCUSSION}

The present study investigated the effects of both horizontal and vertical field of view restrictions on human locomotion through two different complex 3D environments. In particular, we measured the influence of horizontal and vertical field-of-view restrictions on the time needed to traverse courses containing different obstacles. The results show that participants needed significantly more time to complete the courses when their field-of-view was restricted, compared to walking with unrestricted vision. Field-of-view restrictions had same effect for each of the different courses. Hence, the effect is robust, and the results were not characteristic for one particular kind of obstacle, or for the nature of movement required to cross them. 
Proc. SPIE Vol. 6955-16, Head- and Helmet-Mounted Displays XIII: Design and Applications, Orlando FL, USA, March 2008

The results of the second experiment show a significant difference between locomotion performance in the most restricted horizontal field-of-view condition $\left(30^{\circ}\right)$ and that in all other restricted field-of-view conditions. However, there are no significant differences among the other restricted field-of-view conditions. This indicates that the effect of field-of-view restriction on locomotion is not a gradual one, but more like a stepwise relationship. The current results show no further decrease in average walking time for field-of-view sizes larger than $120^{\circ}$, a finding that was unexpected.

A possible explanation may be the fact that participants were free to make head movements. Previous studies have shown that observers tend to compensate for a reduction of their instantaneous visual field by making larger head movements ${ }^{7,9,18,24}$. It appears that the effects of instantaneous field-of-view restrictions can be compensated to some degree through the construction of an "effective field-of-view", that can be obtained by sweeping the instantaneous field-of-view over a larger region of space (i.e. through head movements ${ }^{10}$ ). It has also been observed that loss of peripheral vision results in a decrease of situational awareness, which in turn leads to a decreased confidence ${ }^{1,6}$. Participants may therefore feel less confident when wearing goggles. This may motivate participants to reduce their uncertainty by making large (maximal) compensatory head movements in order to acquire as much visual information as possible. A maximal compensation strategy may explain the identical head movement results for the different field-ofview conditions. The process of gathering additional visual information about the environment by making compensatory head movements may also require additional time, which may explain the reduced manoeuvring speed in restricted field-of-view conditions. A maximal compensation strategy may therefore also explain the fact that no difference in average walking time was found in the range of $75-180^{\circ}$ field-ofview. Yet, at the smallest field-of-view used in this study $\left(30^{\circ}\right.$ horizontal $)$ even the maximal compensation by head movement is not enough to compensate for the adverse effect of field-of-view restriction. Further research is needed to investigate in more detail possible compensatory head motion strategies, probably in combination with eye movements, deployed during locomotion through a complex environment.

There is a significant difference between locomotion performance with each horizontal field-of-view restriction tested in this study and with the unrestricted field-of-view. This holds even for the $180^{\circ}$ field-ofview condition, which is almost equal to a full horizontal field-of-view of about $200^{\circ}$. This effect is probably caused by the vertical field-of-view restriction.

\section{CONCLUSIONS}

The fact that we found no performance degradation over the range of $75-180^{\circ}$ field-of-view in the second experiment may have implications for the development of head-mounted displays and other field-of-view restricting devices that are used in combination with human locomotion tasks. Because we found that fieldof-views in the range of $75-180^{\circ}$ yielded the same performance, it may not be necessary to make the horizontal field-of-view larger than $75^{\circ}$ for this type of locomotion tasks. This would reduce the production costs, and would contribute positively to the ergonomic aspects (higher resolution, less weight, less chance of motion sickness sensations).

\section{ACKNOWLEDGMENT}

This research has been supported by the GATE project, funded by the Netherlands Organization for Scientific Research (NWO) and the Netherlands ICT Research and Innovation Authority (ICT Regie). 
Proc. SPIE Vol. 6955-16, Head- and Helmet-Mounted Displays XIII: Design and Applications, Orlando FL, USA, March 2008

\section{REFERENCES}

1. Alfano, P.L. and Michel, G.F., Restricting the field of view: perceptual and performance effects, Perceptual and Motor Skills, 70(1) ,pp. 35-45, 1990.

2. Amblard, B., Cremieux, J., Marchand, A.R. and Carblanc, A., Lateral orientation and stabilization of human stance: static versus dynamic visual cues, Experimental Brain Research, 61(1),pp. 21-37, 1985.

3. Arthur, K.W. (2000). Effects of field of view on performance with head-mounted displays. Chapel Hill, NC: UNC Chapel Hill.

4. Chevaldonné, M., Ballaz, C., Mérienne, F., Neveu, M., Chevassus, N., Guillaume, F. and Arbez, C., Field-of-view tests for a virtual cockpit application, Journal of Electronic Imaging, 15(2) ,pp. 020501-1-020501-3, 2006.

5. Creem-Regehr, S.H., Willemsen, P., Gooch, A.A. and Thompson, W.B., The influence of restricted viewing conditions on egocentric distance perception: Implications for real and virtual indoor environments, Perception, 34(2), pp. 191-204, 2005.

6. Dolezal, H., Living in a world transformed: perceptual and performatory adaptation to visual distortion, Academic Press, New York, USA, 1982.

7. Gallimore, J.J., Brannon, N.G. and Patterson, F.R., The effects of field-of-view on pilot head movement during low level flight, In: Proceedings of the Human Factors and Ergonomics Society 42nd Annual Meetinga, pp. 6-10, Chicago, IL, 1998.

8. Jennings, S. and Craig, G., Effects of field-of-view on pilot performance in night vision goggles flight trials: preliminary findings, In: R.J. Lewandowski, L.A. Haworth \& H.J. Girolamo (Ed.), Helmetand Head-Mounted Displays V, pp. 335-342, The International Society for Optical Engineering, Bellingham, MA, 2000.

9. Kasper, E., Haworth, L.A., Szoboszlay, Z.P., King, R.D. and Halmos, Z.L., Effects of in-flight fieldof-view restriction on rotorcraft pilot head movement, In: R.J. Lewandowski, L.A. Haworth \& J.J. Girolamo (Ed.), Head-Mounted Displays II, pp. 34-45, The International Society for Optical Engineering, Bellingham, WA, 1997.

10. Knapp, J.M. and Loomis, J.M., Limited field of view of head-mounted displays is not the cause of distance underestimation in virtual environments, Presence: Tele-operators and Virtual environments, 13(5), pp. 572-577, 2004.

11. Latham, R., Head-mounted display survey, Real Time Graphics, 7(2),pp. 8-12, 1999.

12. Lin, J.J.W., Duh, H.B.L., Parker, D.E., Abi-Rached, H. and Furness, T.A., Effects of field of view on presence, enjoyment, memory, and simulator sickness in a virtual environment, In: Proceedings of the IEEE Virtual Reality 2002 (VR.02), pp. 164-171, IEEE Press, Washington, USA, 2002.

13. Loftus, A., Murphy, S., McKenna, I. and Mon-Williams, M., Reduced fields of view are neither necessary nor sufficient for distance underestimation but reduce precision and may cause calibration problems, Experimental Brain Research, 158(3) ,pp. 328-335, 2004.

14. Patterson, R., Winterbottom, M.D. and Pierce, B.J., Perceptual issues in the use of head-mounted visual displays, Human Factors, 48(3) ,pp. 555-573, 2006.

15. Pausch, R., Crea, T. and Conway, M., A literature survey for virtual environments: Military flight simulator visual systems and simulator sickness, Presence: Tele-operators and Virtual environments, 1(3),pp. 344-363, 1992.

16. Psotka, J. \& Lewis, S.A. Effects of field of view on judgements of self-location: distance estimations using plainview representations as a function of observer eye staion points (ESP) abd geometric field of view (FOVg), (Report ARI Research Note 98-24), U.S. Army Research Institute for the Behavioral and Social Studies, Alexandria, Virginia, (1995).

17. RCTA Inc. Minimum operational performance standards for integrated night vision imaging system equipment, (Report DO-275), RCTA Inc., Washington, DC , (2001).

18. Szoboszlay, Z.P., Haworth, L.A., Reynolds, T.L., Lee, A. and Halmos, Z., Effect of field-of-view restriction on rotocraft pilot workload and performance: preliminary results, In: R.J. Lewandowski, W. Stephens \& L.A. Haworth (Ed.), Helmet- and Head-Mounted Displays and Symbology Design 
Proc. SPIE Vol. 6955-16, Head- and Helmet-Mounted Displays XIII: Design and Applications, Orlando FL, USA, March 2008

Requirements II, pp. 142-153, The International Society for Optical Engineering, Bellingham, WA, 1995.

19. Turano, K., Herdman, S.J. and Dagnelie, G., Visual stabilization of posture in retinitis pigmentosa and in artificially restricted visual fields, Investigative Ophthalmology and Visual Science, 34(10) ,pp. 3004-3010, 1993.

20. van Erp, J.B.F. and Padmos, P., Image parameters for driving with indirect viewing systems, Ergonomics, 46(15),pp. 1471-1499, 2004.

21. Wade, L.R., Weimar, W.H. and Davis, J., Effect of personal protective eyewear on postural stability, Ergonomics, 47(15),pp. 1614-1623, 2004.

22. Wagenaar, W.A., Note on the construction of digram-balanced Latin squares, Psychological Bulletin, 72(6) ,pp. 384-386, 1969.

23. Watt, S.J., Bradshaw, M.F. and Rushton, S.K., Field of view affects reaching, not grasping, Experimental Brain Research, 135(3), pp. 411-416, 2000.

24. Wells, M.J. and Venturino, M., Performance and head movements using a helmet-mounted display with different sized fields-of-view, Optical Engineering, 29(8) ,pp. 870-877, 1990.

25. Wells, M.J., Venturino, M. and Osgood, R.K., Effect of field of view size on performance at a simple simulated air-to-air mission, In: J.T. Carollo (Ed.), Helmet-Mounted Displays, pp. 126-130, The International Society for Optical Engineering, Bellingham, WA., USA, 2006.

26. Werner, E.B., Manual of visual fields , Churchill Livingstone, New York, USA, 1991. 